\section{Zika Virus in the Joint of a Patient with Rheumatoid Arthritis}

\section{To the Editor:}

Since 2015, Brazil has experienced a major public health crisis caused by the arboviruses Zika (ZIKV) and Chikungunya (CHIKV), which are circulating along with the dengue virus ${ }^{1}$.

ZIKV is now considered endemic in all Brazilian states and is currently spreading widely in South and Central America and is threatening the United States and Europe ${ }^{2,3}$. ZIKV and CHIKV share similar acute clinical presentation with arthralgia, skin rash, and fever, which also resembles other rheumatic diseases ${ }^{1,4}$. Most relevantly, ZIKV and CHIKV may complicate the clinical condition of rheumatic patients.

We share here the case of a 53-year-old woman with a 4-year diagnosis of rheumatoid arthritis (RA) and who achieved clinical remission with a combined therapy of daily prednisone $(5 \mathrm{mg}$ ) and weekly etanercept (ETN; $50 \mathrm{mg}$ ) and methotrexate (MTX; $20 \mathrm{mg}$ ) 4 months before the clinical event described below.

We obtained the patient's written informed consent to publish the material and have received the ethics board approval of the Hospital Universitario Clementino Fraga Filho da UFRJ - CEP (comitê de ética em pesquisa; number: 1.541.752).

The patient presented with fever $\left(37.8^{\circ} \mathrm{C}\right)$; a maculopapular rash of the face, trunk, and limbs; bilateral conjunctivitis (Figure 1); and arthritic inflammation of the fingers, wrist, right knee, and ankles. She reported that her neighbors had similar symptoms. ETN and MTX were suspended, and
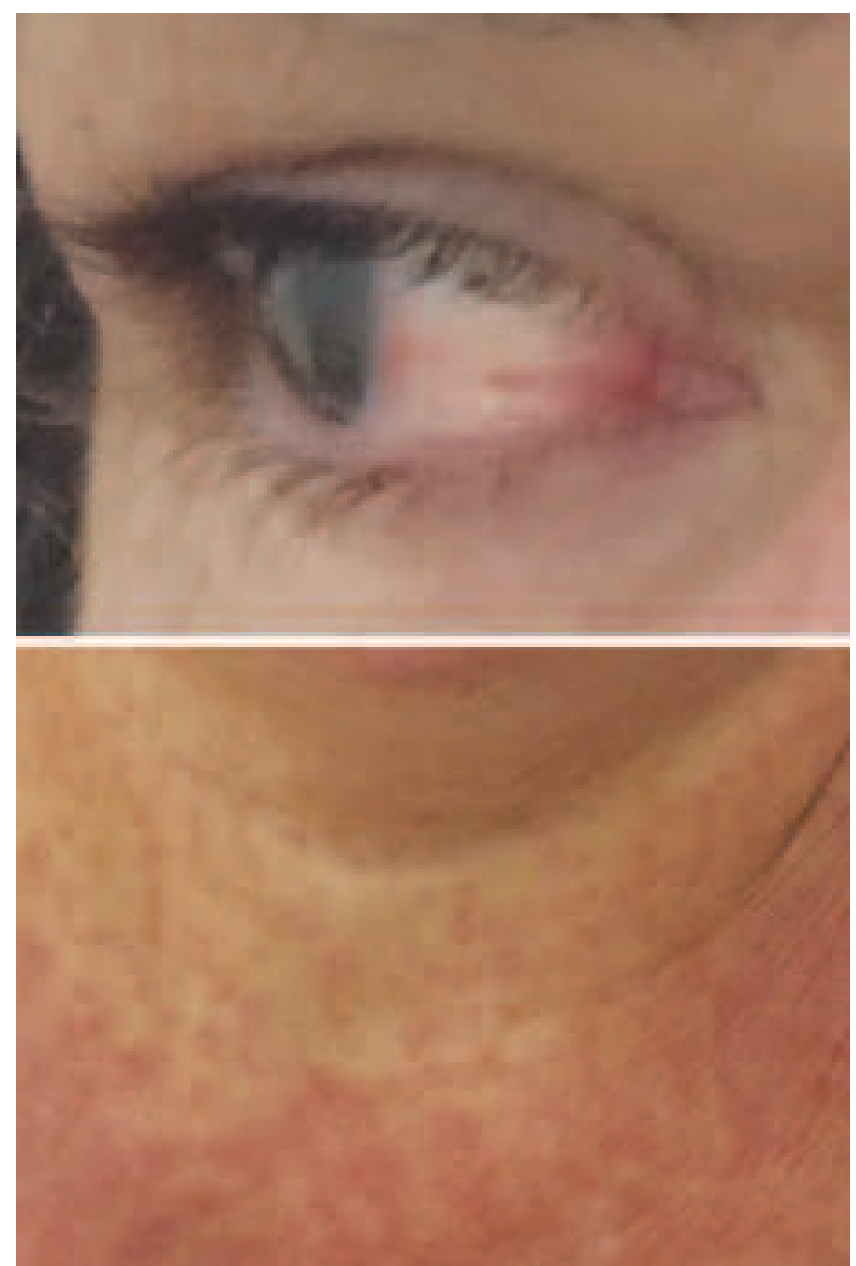

Figure 1. Zika infection in patient with rheumatoid arthritis. Conjunctivitis and maculopapular rash. blood and knee synovial fluid (SF) were collected for molecular testing ${ }^{5,6}$ for the ZIKV and CHIKV viruses ${ }^{3}$. Only ZIKV was identified in both samples by real-time PCR. Her fever lasted 2 days, the conjunctivitis lasted 4 days, and rash and arthritis showed improvement on the fifth day. At Day 7, symptoms had disappeared and a followup blood sample was negative for ZIKV by molecular test. Dengue virus-specific identification immunoglobulin $\mathrm{M}(\operatorname{IgM})$ and indirect IgG ELISA assays (Standard Diagnostic Inc.) performed with a sample of that day were negative for IgM and positive for $\mathrm{IgG}$, consistent with a prior (over $10 \mathrm{yrs}$ ) dengue illness.

Thirteen days after her first visit, the patient returned to the clinic, complaining of mild arthralgia and minor effusion of the right knee. New blood and SF samples were collected and tested for ZIKV and CHIKV. Again, only ZIKV was found in the SF, but it remained negative in the blood. ZIKV RNA fragments ( 843 base pairs) isolated from the patient's blood and SF samples were subjected to PCR sequencing, and no differences were found between them. The sequence was compared to all sequences generated in the laboratory to rule out cross-contamination and to confirm uniqueness. These findings characterized for the first time an infection caused by ZIKV within the joint of a patient. Although the reasons for the presence of ZIKV in the SF are unknown, it may be that the patient's RA facilitated viral access to the joint cavity. ZIKV in SF after it has been cleared from the circulating blood may be associated with prolonged arthralgia and may provide a repository for viral replication. This case broadens the spectrum of ZIKV pathogenesis, raises concerns regarding additional dangers of ZIKV in patients with RA or other autoimmune diseases, and highlights the need for further studies on the appropriate use of immunosuppressive therapies in this scenario.

LUIS ROIMICHER, MD, PhD, Professor of Rheumatology, Department of Rheumatology-Internal Medicine, Hospital Universitário CFF, Universidade Federal do Rio de Janeiro; ORLANDO C. FERREIRA JR., MD, PhD, Professor, Laboratory of Molecular Virology, Centro de Ciências da Saúde, Universidade Federal do Rio de Janeiro; MONICA B. ARRUDA, PhD, Professor, Laboratory of Molecular Virology, Centro de Ciências da Saúde, Universidade Federal do Rio de Janeiro; AMILCAR TANURI, MD, PhD, Laboratory of Molecular Virology, Centro de Ciências da Saúde, Universidade Federal do Rio de Janeiro, Rio de Janeiro, Brazil. Address correspondence to Dr. L. Roimicher, Department of Rheumatology-Internal Medicine, Hospital Universitário CFF, Universidade Federal do Rio de Janeiro, Rua Prof Rodolpho Paulo Rocco 255, 70 Andar, Rio de Janeiro, Brazil.

E-mail: mail-luizroimicher@gmail.com

\section{REFERENCES}

1. Brasil P, Calvet GA, Siqueira AM, Wakimoto M, de Sequeira PC, Nobre A, et al. Zika virus outbreak in Rio de Janeiro, Brazil: clinical characterization, epidemiological and virological aspects PLoS Negl Trop Dis 2016;10:e0004636.

2. Akiner MM, Demirci B, Babuadze G, Robert V, Schaffner F. Spread of the invasive mosquitoes Aedes aegypti and Aedes albopictus in the Black Sea region increases risk of chikungunya, dengue, and Zika outbreaks in Europe. PLoS Negl Trop Dis 2016;10:e0004664.

3. Fauci AS, Morens DM. Zika virus in the Americas-yet another arbovirus threat. N Engl J Med 2016;374:601-4.

4. Chang C, Ortiz K, Ansari A, Gershwin ME. The Zika outbreak of the 21st century. J Autoimmun 2016;68:1-13.

5. Lanciotti RS, Kosoy OL, Laven JJ, Velez JO, Lambert AJ, Johnson $\mathrm{AJ}$, et al. Genetic and serologic properties of Zika virus associated with an epidemic, Yap State, Micronesia, 2007. Emerg Infect Dis 2008;14:1232-9.

6. Lanciotti RS, Kosoy OL, Laven JJ, Panella AJ, Velez JO, Lambert $\mathrm{AJ}$, et al. Chikungunya virus in US travelers returning from India, 2006. Emerg Infect Dis 2007;13:764-7.

J Rheumatol 2017;44:4; doi:10.3899/jrheum.160722 